\title{
The thermal neutron capture cross section of ${ }^{129}$ I
}

\author{
T. Belgya ${ }^{1, a}$, O. Bouland ${ }^{2}$, G. Noguere ${ }^{2}$, A. Plompen ${ }^{3}$, P. Schillebeeckx ${ }^{3}$, and L. Szentmiklósi ${ }^{1}$ \\ Institute of Isotopes Hungarian Academy of Sciences, P.O. Box 77, 1525 Budapest, Hungary \\ 2 CEA Cadarache, DEN/CAD/DER/SPRC/LEPh, 13108 Saint-Paul-lez-Durance, France \\ ${ }^{3}$ EC-JRC, IRMM, Neutron Physics Unit, 2440 Geel, Belgium
}

\begin{abstract}
The thermal neutron capture cross section of ${ }^{129}$ I Long Lived Fission Product is determined from chopped cold neutron beam experiments preformed at the PGAA facilities of the Institute of Isotopes. The new experimental value is more precise than the earlier values and is in agreement with recent results of Nakamura et al. and fits well to the differential cross section measurements of Noguere et al. A detailed description of the analysis is given.
\end{abstract}

\section{Introduction}

In the radioactive waste management one of the most important Long Lived Fission Product (LLFP) is ${ }^{129} \mathrm{I}$. The $\beta^{-}$decay half-life of ${ }^{129} \mathrm{I}$ is $1.57 \times 10^{7} \mathrm{a}$. It represents a substantial amount of the total radio toxicity of LLFP [1] because of its mobility in the environment [2]. The thermal neutron capture cross section of ${ }^{129} \mathrm{I}$ is an important quantity to determine the transmutation efficiency in various transmutation facilities such as Accelerator Driven System (ADS) [3,4]. Recently a series of experiments was performed at the GELINA facilities of the JRC Institute for Reference Materials and Measurements (IRMM) to determine the capture cross sections of ${ }^{127} \mathrm{I}$ and ${ }^{129} \mathrm{I}$ in the resonance region (RR). Detailed analyses of the experimental time of flight spectra were described in ref [5]. To perform the low energy RR analyses of ${ }^{127} \mathrm{I}$ and ${ }^{129} \mathrm{I}$, the thermal neutron capture cross section point is used to set the parameters of the negative resonance. This requires a rather precise value for the thermal neutron cross section.

In the literature the measured cross section values scatter between $24-35$ b for ${ }^{129} \mathrm{I}$. A part of these cross sections were determined a long time ago with various methods [6-10]. A valuable study of the radioactivity of ${ }^{130 \mathrm{~g}, \mathrm{~m}} \mathrm{I}$ was performed by Hopke et al. [11] with different reactions. They determined a very precise half-life of the ${ }^{130}$ I ground state beta-decay to be 12.36(1) h. Samples were irradiated at the MIT reactor. From the growth and decay characteristics of the prominent gammatransitions, the value of $0.661(80)$ was obtained for the ratio of ground-state to metastable-state (n, $\gamma$ ) cross sections, which differs from the value of 0.5 of Wilkey and Willard [12]. It is not clear, however, whether the epithermal neutrons have or have not influenced this ratio. More recently Nakamura et al. [13] repeated the activation of ${ }^{129}$ I at the TRIGA MARKII reactor of the Rikkyo University and determined the thermal neutron capture cross section and the resonance integral. They found a $4.3 \%$ smaller half-life for the isomer than was given in the Table of Isotopes [14], but they agreed with the ground state half life of Hopke et al. [11]. They obtained a value for the isomer decay branching ratio to the ground state of

${ }^{a}$ Presenting author, e-mail: Belgya@iki.kfki.hu
82.3(4)\%, which agrees with the data published by Hopke et al. [11], but differs from Wilkey and Willard [12]. For the ground state to isomer cross section ratio Nakamura et al. obtained a value of $0.702(40)$.

To add more independent values, a series of experiments was performed at the Budapest PGAA-NIPS facilities to determine the cross sections using a target containing ${ }^{127} \mathrm{I}$ and ${ }^{129} \mathrm{I}$ which was provided and prepared [2] by IRMM. The target contained both ${ }^{127} \mathrm{I}$ and ${ }^{129} \mathrm{I}$ in $\mathrm{PbI}_{2}$ chemical form with a precisely determined mass ratio. The well known mass ratio made it possible to determine the thermal neutron capture cross section of ${ }^{129}$ I using the partial gamma-ray production cross section of the ${ }^{128}$ I $443 \mathrm{keV}$ decay gamma-ray, which was also produced in our neutron capture experiments.

In a subsequent series of experiments, a new beam chopper based method [15] made it possible to carry out an in-beam cyclic activation to determine the partial gamma-ray production cross section $\sigma_{443}$ of the $443 \mathrm{keV}$ transition following the beta decay of ${ }^{128} \mathrm{I}$ produced in the ${ }^{127} \mathrm{I}(\mathrm{n}, \gamma)^{128} \mathrm{I}$ reaction. The $\sigma_{443}$ could be determined with higher precision than from our earlier continuous neutron beam experiments [16], mainly due to the much simpler spectrum in the beam-off time. This new $\sigma_{443}$ value was used as internal comparator [17] in the ${ }^{129} \mathrm{I}$ capture experiments. The chopped beam technique reduces significantly the possible sources of uncertainties such as the knowledge of flux-inhomogenity and energy distribution, and the neutron absorption in the target. Since the applied cold neutron beam has negligible amount of epithermal neutrons, and both nuclides have cross sections of $1 / v$ type, the obtained cross sections can be converted in thermal equivalent values which are free of the disturbing resonance capture contribution [18]. In the following sections these chopper experiments and data evaluation are described and compared to the literature.

\section{Experiments}

A ${ }^{129}$ I containing sample was received from IRMM with a lot number of $9008 \mathrm{~F}$. The $1.9778 \mathrm{~g}$ sample was sealed in an aluminum vial of $1.6 \mathrm{~cm}$ diameter. The isotopic composition of the sample was determined in a series of experiments 
performed at the analytical chemistry laboratory of the Paul Scherrer Institute (PSI) and at IRMM with mass spectrometers, the $7 \mathrm{MeV}$ van de Graaff accelerator and Neutron Resonance Transmission Analysis at Gelina [19]. The target contained iodine $(20 \mathrm{w} \%)$, lead $(53 \mathrm{w} \%)$, sulfur $(6 \mathrm{w} \%)$, sodium ( $1 \mathrm{w} \%)$, oxygen $(15 \mathrm{w} \%)$, hydrogen $(0.1 \mathrm{w} \%)$ and nitrogen $(1 \mathrm{w} \%)$ approximately. This composition could be used to determine gamma-ray absorption. The total activity of the sample was $2.81 \mathrm{MBq}$.

All experiments were performed at the cold-neutron beam PGAA facility [20] of the Budapest Research Reactor. The gamma-ray spectrometer was a $23 \%$ HPGe detector with a BGO annulus, which was operated in Compton-suppression mode. The $23 \mathrm{~cm}$ detector to sample distance was sufficiently large to avoid any disturbing true coincidence effects.

The most recent set of experiments with the ${ }^{129}$ I containing sample was performed at the PGAA facility using a chopped beam [15,21]. The beam was collimated to $23 \mathrm{~mm}^{2}$ in order to decrease the dead time during the activation. The average dead time was approximately $11 \%$ during the beam-on phase and less than $1 \%$ in the beam-off phase. Since the comparator peak and the unknown intensity peak are both decay peaks it is advantageous to use only the beam-off phase to determine the ${ }^{129}$ I partial gamma-ray production cross sections because the decay spectrum is much simpler than the prompt gamma spectrum. Four spectra were stored in one continuous choppedbeam activation measurement at measuring times of $6181 \mathrm{~s}$, $81836 \mathrm{~s}, 93056 \mathrm{~s}$ and $100730 \mathrm{~s}$. A ${ }^{207} \mathrm{Bi}$ source put in the direct view of the PGAA detector served as dead time correction for the spectrometer. The prompt and decay spectra stored at $100730 \mathrm{~s}$ are compared in figure 1.

Figure 1 clearly shows a well-isolated $443 \mathrm{keV}$ comparator peak of ${ }^{128} \mathrm{Xe}$ in the decay-phase spectrum in the enlarged insert.

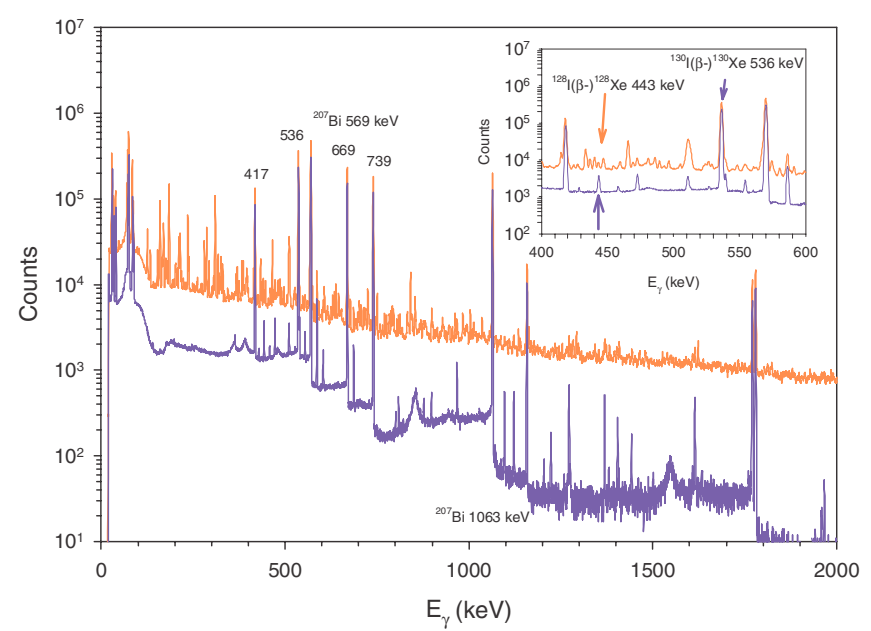

Fig. 1. The prompt-(upper curve) and the decay-phase (lower curve) chopper spectra without normalization. The most intense decay peaks of ${ }^{130} \mathrm{I}\left(\beta^{-}\right)^{130} \mathrm{Xe}$ are labeled with their energy in $\mathrm{keV}$. The two prominent ${ }^{207} \mathrm{Bi}$ source lines are also labeled. The insert shows the $400-600 \mathrm{keV}$ region where the comparator $443 \mathrm{keV}{ }^{128} \mathrm{I}\left(\beta^{-}\right)^{128} \mathrm{Xe}$ gamma ray is standing alone in the decay spectrum, while in the prompt spectrum it is the smallest member of a multiplet of gamma rays.

\section{Determination of the ${ }^{129}$ I capture cross section}

The cross section determination of ${ }^{129} \mathrm{I}$ from our chopped beam experiments is based on the formula (2) and the methodology given in Szentmilósi et al. [15]. Here we introduce additional correction term, which accounts for the neutron and gamma absorption of the target as well. The modified formula for the measured gamma-ray peak area $A_{d}$ in the decay phase is

$$
A_{d}=\sigma_{d} \phi n_{d} \varepsilon\left(E_{d}\right) K L t_{m} \eta_{d} B\left(t_{m}\right) f\left(E_{d}\right)
$$

where $\sigma_{d}$ is the partial gamma-ray production cross section of the daughter isotope created in the capture reaction. The number $\phi$ is the neutron flux irradiating the sample, $n_{d}$ is the number of target atoms and $\varepsilon\left(E_{d}\right)$ is the gamma-ray detector efficiency at energy $E_{d} . K$ is the proportion of the irradiation time ( $K=0.5)$, while $L$ is the proportion of the decay counting time to the length of a complete chopper cycle, $t_{m}$ is the total measuring time and $\eta_{d}$ accounts for the dead time losses. $B\left(t_{m}\right)$ is the so-called in-beam saturation factor. The $f\left(E_{d}\right)$ factor accounts for the neutron and gamma-ray attenuation in the sample. This later is calculated with numerical integration using a first order approximation for neutron scattering and absorption weighted by the gamma attenuation in the sample. The ${ }^{129}$ I partial gamma-ray cross sections are determined from the comparator technique as described by Révay et al. [17], however using equation (1) from which we drop index $d$ because both the unknown and comparator are in the decay spectrum.

$$
\sigma_{X}=\sigma_{C} \frac{A_{X}}{A_{C}} \frac{n_{C} \varepsilon\left(E_{C}\right) B_{C} f\left(E_{C}\right)}{n_{X} \varepsilon\left(E_{X}\right) B_{X} f\left(E_{X}\right)} .
$$

The gamma ray of $443 \mathrm{keV}$ from the ${ }^{127} \mathrm{I}(\mathrm{n}, \gamma)^{128} \mathrm{I}\left(\beta^{-}\right)^{128} \mathrm{Xe}$ reaction is used as the comparator. The partial gamma-ray cross section of $443 \mathrm{keV}$ has been recently re-determined with the same chopper technique by Szentmiklósi et al. [15]. Its new value is $0.712(9) \mathrm{b}$ which is slightly higher than the value of $0.678(11) \mathrm{b}$ that could be determined from the continuous neutron beam experiments [16]. This new value can be converted to $\mathrm{k}_{0}$ value relative to the gold comparator and it yields $0.0117(2)$, which agrees fairly well with the 0.0112(2) published by De Corte and Simonits [22].

From here we give detailed description of ratios in equation (2). The peak area ratio $A_{X} / A_{C}$ obtained from the Hypermet fit of the longest measured spectrum is 1189090(1338)/ $11552(170)=102.9(15)$.

The ratio of the number of comparator ${ }^{127} \mathrm{I}$ and the ${ }^{129} \mathrm{I}$ atoms $\left({ }^{127} \mathrm{I} /{ }^{129} \mathrm{I}\right)$ were determined from several experiments with ICP-MS, activation and neutron resonance capture methods at IRMM. The two most precise values were obtained from two different ICP-MS experiments at IRMM [19]. The ${ }^{127} \mathrm{I} /{ }^{129} \mathrm{I}$ values of $0.2088(52)$ and $0.2012(49)$ were obtained by inversion from the values given in ref. [19]. An additional ICPMS result of 0.2035(13) was obtained from the Paul Scherer Institute. Because of the difficulties in describing uncertainties discussed by Noguere et al. [19], we decided to use the unweighted mean value of the three ratios:

$$
\frac{n_{C}}{n_{X}}=\sum_{i=1}^{3}\left({ }^{127} I /{ }^{129} I\right)_{i} / 3 .
$$




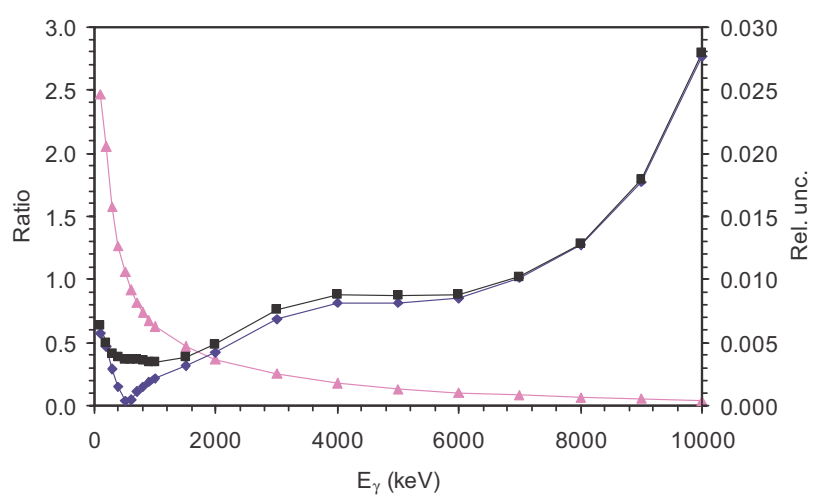

Fig. 2. The efficiency ratio of $\varepsilon\left(E_{\gamma}\right) / \varepsilon(536)$ (left axis) as a function of the comparator energy (triangle). The correlated (diamond) and uncorrelated (square) relative uncertainties (right axis). The lines just connect the calculated points.

The uncertainty of the average given in equation (3) were taken to be the standard deviation. These calculations yield a value of $0.2045(38)$ for the ${ }^{127} \mathrm{I} /{ }^{129} \mathrm{I}$ isotopic ratio.

The efficiency ratio was determined from our regular efficiency measurements using the evaluation technique described by Molnár et al. [23]. The uncertainty of the ratio was determined according to the prescription of Révay [24], which applies the correlation between efficiency values at different energies that result from a least-squares fit. Due to the close energy of the comparator 443-keV gamma ray to the unknown cross section 536-keV gamma ray, the correlation introduces negligible uncertainty in their efficiency ratio. The importance of the correlation is demonstrated in figure 2.

The value for the $\varepsilon(436) / \varepsilon(536)$ ratio is $1.163(1)$. As expected the uncertainty of the efficiencies ratio is negligibly small, being less than $0.1 \%$.

The saturation factors depend on the pathways of the beta-decay processes. While $6.3 \%$ of ${ }^{128} \mathrm{I}$ decays to ${ }^{128} \mathrm{Te}$ with electron conversion, the more intense decay path is the beta decay to ${ }^{128} \mathrm{Xe}$. The most intense $443-\mathrm{keV}$ gamma ray (the comparator) in the latter case follows the type-I decay according to the classification De Corte and Simonits [22]. The type-I in-beam saturation factor has the form of

$$
B_{C}=1-\frac{1-\exp \left(-\lambda_{C} t_{m}\right)}{\lambda_{C} t_{m}}
$$

where $\lambda_{C}$ is the decay constant of ${ }^{128}$ I ground state. For our cyclic activation experiment $B_{C}$ can be written as

$$
B_{C C}=B_{C} L K=\left(1-\frac{1-\exp \left(-\lambda_{C} t_{m}\right)}{\lambda_{C} t_{m}}\right) \frac{t_{a}}{T} \frac{t_{c}}{T},
$$

where $t_{a}$ and $t_{c}$ are the activation and counting time respectively in a complete revolution time $T$ of the chopper. The saturation factor for the chopped beam activation for the ${ }^{129} \mathrm{I}(\mathrm{n}, \gamma){ }^{130} \mathrm{I}\left(\beta^{-}\right){ }^{130} \mathrm{Xe}$ process can be obtained using the decay scheme in figure 3 .

This decay scheme contains two type-I and one type-II decay-paths. The corresponding chopped in-beam saturation

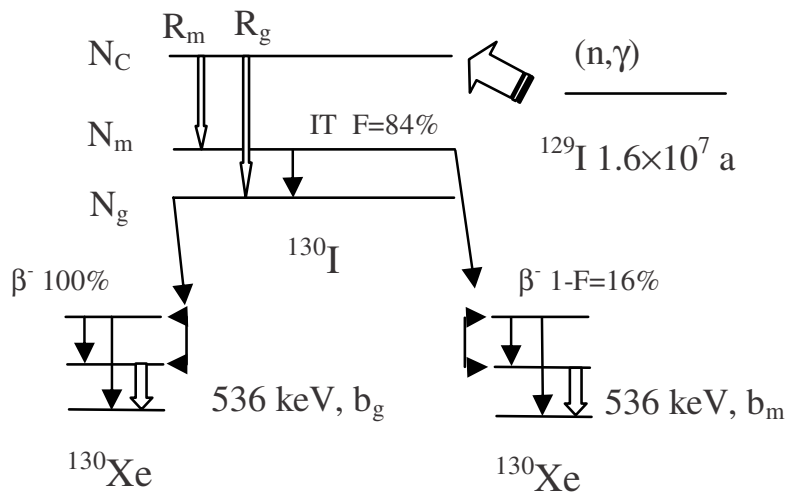

Fig. 3. Decay scheme of the ${ }^{129} \mathrm{I}(\mathrm{n}, \gamma)^{130} \mathrm{I}\left(\beta^{-}\right)^{130} \mathrm{Xe}$ process.

Table 1. Parameters of the ${ }^{129} \mathrm{I}(\mathrm{n}, \gamma)^{130} \mathrm{I}\left(\beta^{-}\right)^{130}$ Xe reaction process.

\begin{tabular}{ll}
\hline$R_{m}$ & $0.588(14)$ \\
$\lambda_{m}$ & $1.3158(14) \times 10^{-3} \mathrm{~s}^{-1}$ \\
$\lambda_{g}$ & $1.5603(24) \times 10^{-5} \mathrm{~s}^{-1}$ \\
$F$ & $0.823(3)$ \\
$b_{g}$ & 0.997 \\
$b_{m}$ & 0.988 \\
\hline
\end{tabular}

factor can be written in the following form

$$
\begin{aligned}
& B_{X C}=L K\left\{\begin{array}{l}
b_{g} R_{g} B_{I}\left(\lambda_{g}\right)+b_{m} R_{m}(1-F) B_{I}\left(\lambda_{m}\right) \\
+b_{g} R_{m} F B_{I I}\left(\lambda_{m}, \lambda_{g}\right)
\end{array}\right\} . \\
& \left(b_{g} R_{g}+b_{m} R_{m}(1-F)+b_{g} R_{m} F\right)^{-1}
\end{aligned}
$$

To calculate the $B_{C C}$ and $B_{X C}$ saturation-factor ratio we decided to use the values from the Table of Isotopes [25] and from the article of Nakamura et al. [13] (see table 1). It is important to note that when the ratio is calculated the $\mathrm{L}$ and $\mathrm{K}$ factors cancel. It is only true when both the comparator and unknown are decay gamma rays.

It turned out that many different correlated parameter value sets reproduce our measured activation and decay curves equally well; thus they require more thorough studies, and furthermore these rather different sets of values hardly change the resulting $B_{X C}$ values. The $B_{C C} / B_{X C}$ value with the parameters of table 1 is 1.803(40) (1.805 with our best-fit decayparameters) for the $100730 \mathrm{~s}$ long experiment. The resulting uncertainty is estimated to be about $2 \%$ from the uncertainty of the branching ratio $R_{m}$, which dominates the uncertainty of the $B_{C C} / B_{X C}$ ratio.

The correction due to the attenuation of neutrons and gamma-ray self absorption of the target for the 443-keV and the 536-keV gamma ray is $f\left(E_{C}\right)=0.872$ and $f\left(E_{X}\right)=0.901$ respectively. The relative uncertainty of the ratio is estimated to be smaller than $0.5 \%$ because it behaves similarly to the correlated efficiency ratios. The calculated value is $0.968(5)$.

The partial gamma ray production cross section of $536 \mathrm{keV}$ can be obtained from equation (2) using the above given factors. The resulting saturation value is $\sigma_{536}=30.4(11) \mathrm{b}$. To calculate the thermal capture cross section we have to take into the account the conversion factor and the saturation branching ratio of the 536-keV gamma-ray as well

$$
\sigma_{t h}=\sigma_{536}\left(1+\alpha_{536}\right) / P_{\gamma},
$$


where $\alpha_{536}$ is the internal conversion coefficient and the value of the branching ratio $P_{\gamma \mathrm{g}}\left(=b_{g} R_{g}+b_{m} R_{m}(1-F)+b_{g} R_{m} F\right)$ is calculated to be 0.996 with negligible uncertainty. The value of $\alpha_{536}$ is 0.00748 , which was taken from the Table of Isotopes [25]. The thermal neutron capture cross section of ${ }^{129} \mathrm{I}$ is $\sigma_{\text {th }}=30.6(11)$.

Our new value agrees well with the most recent value of Nakamura et al. [13] and is also within one or two sigma values from the older results [6-10]. To arrive at an evaluated final recommended value a more thorough comparison is necessary.

In a summary, we can say that the decay parameters of the ${ }^{129} \mathrm{I}(\mathrm{n}, \gamma)^{130} \mathrm{I}\left(\beta^{-}\right){ }^{130} \mathrm{Xe}$ reaction process should be remeasured. Our best fit of these parameters differs quite a lot from the parameters published by Nakamura et al. [13], but it does not influence the in-beam saturation factor for longer measurement times (much longer than the isomer halflife), so the obtained thermal neutron cross section can be considered as our final value. The chopped beam measurement substantially improved the signal-to-background ratio relative to our earlier continuous beam measurements and gave us better confidence in our new thermal cross section value. Our new partial gamma ray cross section for the $536 \mathrm{keV}$ transition in ${ }^{130} \mathrm{Xe}$ can be used in activation analysis after conversion to $\mathrm{k}_{0}$ value based on the gold standard.

This work was supported by the NAP VENEUS05 No. OMFB 00184/2006, the GVOP-3.2.1.-2004-04-0268/3 and the EU FP6 NMI3 No. RIII-CT-2003-505925 projects.

\section{References}

1. S. Gordelier, C. Nordborg, C. Pescatore, NEA News 24, 7 (2006).

2. C. Ingelbrecht, J. Lupo, K. Raptis, T. Altzitzoglou, G. Noguere, Nucl. Instrum. Meth. A 480, 204 (2002).

3. A. Ab'anades, J. Aleixandre, S. Andriamonje, A. Angelopoulos, A. Apostolakis, H. Arnould, E. Belle, C.A. Bompas, D. Brozzi, J. Bueno et al., Nucl. Instrum. Meth. A 478, 577 (2002).

4. A. Stanculescu, Emerging nuclear energy and transmutation systems: Core physics and engineering aspects, IAEA, TECDOC1356, Vienna (2003).
5. G. Noguere, O. Bouland, A. Brusegan, P. Schillebeeckx, P. Siegler, A. Lepretre, N. Herault, G. Rudolf, Phys. Rev. C 74, 054602-1-19 (2006).

6. R.C. Block, G.G. Slaughter, J.A. Harvey, Nucl. Sci. Eng. 8, 112 (1960).

7. J.C. Roy, D. Wuschke, Can. J. Chem. 36, 1424 (1958).

8. B.C. Purkazastha, G.R. Martin, Can. J. Chem. 34, 293 (1956).

9. N.J. Pattenden, Nucl. Sci. Eng. 17, 371 (1963).

10. L. Friedmann, D.C. Aumann, Radiochimica Acta 33, 182 (1983).

11. P.K. Hopke, A.G. Jones, W.B. Walters, A. Prindle, R.A. Mezer, Phys. Rev. C 8, 745 (1973).

12. D.D. Wilkey, J.E. Willard, J. Chem. Phys. 44, 970 (1966).

13. S. Nakamura, H. Harada, T. Katoh, Y. Ogata, J. Nucl. Sci. Technol. 33, 283 (1996).

14. C.M. Lederer, V.S. Shirley, Table of isotopes (John Wiley \& Sons, 1978)

15. L. Szentmiklósi, Zs. Révay, T. Belgya, Nucl. Instrum. Methods A 564, 655 (2006).

16. T. Belgya, G.L. Molnár, Zs. Révay, J. Weil, 10th International Conference on Nuclear Data for Science and Technology, Sept. 26-Oct. 1, 2004, Santa Fe, New Mexico (2005), pp. 744747.

17. Zs. Révay, G.L. Molnár, Radiochimica Acta 91, 361 (2003).

18. R.M. Lindstrom, Zs. Révay, Beams and Facilities (Kluwer Academic Publishers, Dordrecht, Boston, London, 2004), pp. 31-58.

19. G. Noguere, F. Cserpák, C. Ingelbrecht, A.J.M. Plompen, C.R. Quetel, P. Schillebeeckx, Nucl. Instrum. Meth. A (2007), (in press).

20. Zs. Révay, T. Belgya, Zs. Kasztovszky, J.L. Weil, G.L. Molnár, Nucl. Instrum. Meth. B 213, 385 (2004).

21. Zs. Révay, T. Belgya, L. Szentmiklósi, G.L. Molnár, J. Rad. Nucl. Chem. 264, 277 (2005).

22. F. De Corte, A. Simonits, At. Data Nucl. Data Tables 85, 47 (2003).

23. G.L. Molnár, Zs. Révay, T. Belgya, Nucl. Instrum. Meth. Phys. Res. Sect. A-Accelerators Spectrometers Detectors and Associated Equipment 489, 140 (2002).

24. Zs. Révay, Nucl. Instrum. Meth. A 564, 688 (2006).

25. R.B. Firestone, C.M. Baglin, S.Y.F. Chu, Table of Isotopes (Wiley, 1999). 